Research Square
Preprints are preliminary reports that have not undergone peer review.

They should not be considered conclusive, used to inform clinical practice, or referenced by the media as validated information.

\title{
Methodological Perspectives on the Study of the Health Effects of Unemployment - Reviewing the Mode of Unemployment, the Statistical Analysis Method and the Role of Confounding Factors
}

Fredrik Norström ( $\square$ fredrik.norstrom@umu.se)

Umeå University

Anne Hammarström

Karolinska Institutet

\section{Research Article}

Keywords: health, unemployment, statistical analysis, confounding

Posted Date: December 13th, 2021

DOI: https://doi.org/10.21203/rs.3.rs-1123592/v1

License: (c) (i) This work is licensed under a Creative Commons Attribution 4.0 International License. Read Full License 


\section{Abstract \\ Introduction:}

Studying the relationship between unemployment and health raises many methodological challenges. In the current study, the aim was to evaluate how different ways of measuring unemployment and the choice of statistical model affects the effect estimate.

\section{Methods:}

The Northern Swedish cohort was used, and two follow-up surveys thereof from 1995 and 2007, as well as register data about unemployment. Self-reported current unemployment, self-reported accumulated unemployment and register-based accumulated unemployment were used to measure unemployment and its effect on self-reported health was evaluated. Analyses were conducted with G-computation, logistic regression and three estimators for the inverse probability weighting propensity scores, and 11 potentially confounding variables were part of the analyses. Results were presented with absolute differences in the proportion with poor self-reported health between unemployed and employed individuals for all estimators but logistic regression.

\section{Results:}

Of the initial 1083 pupils in the cohort, 488-693 individuals were defined as employed and 61-214 individuals were defined as unemployed in our different analyses. In the analyses, the deviation was large between the unemployment measures, with a difference of at least $2.5 \%$ in effect size when unemployed was compared with employed for the self-reported and register-based unemployment modes. The choice of statistical method only had a small influence on effect estimates and the deviation was in most cases lower than $1 \%$. When models were compared based on the choice of potential confounders in the analytical model, the deviations were rarely above $0.6 \%$ when comparing models with 4 and 11 potential confounders. Our variable for health selection was the only one that strongly affected estimates when it was not part of the statistical model.

\section{Conclusions:}

Misspecifications of the statistical model or choice of analytical method might not matter much for effect estimates of the relationship between unemployment and health except for the inclusion of a variable measuring earlier health status before becoming unemployed. On the other hand, how unemployment is measured is highly important.

\section{Introduction}

Several studies have shown that unemployment is linked to deteriorated health [1, 2]. For studies on the health effect of unemployment, several methodological issues need to be handled. In this study, we aim to investigate the key components of the analyses, including how unemployment is measured, by responding to slightly different research questions, the statistical analysis method and the variables that are included in analyses to improve estimates. In this study, we use current self-reported unemployment, and recent unemployment, both with self-reported and register-based information, as modes of unemployment. Current unemployment differs from the other modes in that it does not take accumulated exposure into account, while self-reported and register-based information can differ in many ways. Register-based unemployment is often seen as more objectively measured, while self-reported unemployment can be seen as more validly measured. Furthermore, self-reported data might not include shorter periods of unemployment between jobs, which is reported in register data. On the other hand, register data only include the number of days that people are registered as unemployed, and therefore lack information about unemployment when people are not entitled to unemployment benefits.

In a previous methodological review article of publications relating to unemployment and health, it was concluded that there were major weaknesses in many reporting aspects [3]. It was also reported that some of the reviewed manuscripts had probably used a statistical analysis method poorly fitted to the research question. A poor choice of the statistical analysis model could lead to biased results and consequently wrong conclusions [4]. It is therefore extremely important to understand whether data are behaving according to the chosen method. Hence the motivation to use a thorough evaluation in this study.

To achieve an estimate of the causal effect with a small bias in observational studies, a well-designed study is required with a well-defined research question and a well-adapted statistical analysis method. Well-defined exposure(s) and outcome(s) are essential; however, other variables that can play an essential role in the analysis are also crucial. For additional variables to contribute to the model, they need to confound the relation between exposure and outcome, and they should be chosen ahead of the analyses. In this study, the choice of the statistical analysis model is evaluated, as well as how sensitive results are for the selection of additional variables in the analysis. The key confounding factor to handle when unemployment and health are studied is previous health, as the risk of becoming unemployed also depends on health [5], usually referred to as health selection to unemployment.

Within the field of unemployment and health, logistic regression is the most common statistical method, but other regression techniques are also common [1]. Despite the popularity of logistic regression, it seems that awareness of its limitations is generally poor, which is likely to produce highly biased estimates [3, 6]. Methods based on so-called propensity scores, introduced in 1983 by Rosenbaum and Rubin [7], and G-computation have scarcely been used within public health [8], including our field of interest [1, 9]. Propensity score methods and traditional regression modelling, such as G-computation, tend to yield similar results [10], but this has yet to be investigated within our field of interest. 
Our main aim is to evaluate how effect estimates differ based on the mode of unemployment and the choice of statistical model when the relationship between unemployment and health is studied.

\section{Methods}

\section{Study design and participants}

In our study, the Northern Swedish cohort was used [11], and our analyses are carried out on the same data set as was used in a previous publication, which showed that unemployment has a long-term negative health effect for those unemployed during young adulthood [9].

The Northern Swedish cohort was initiated in 1981, with all 1083 pupils in the last year of compulsory school during this year in a middle-sized town in Northern Sweden invited to participate in a study. Repeated questionnaires have been completed, including a matrix with detailed questions about labour market history since the last follow-up [12], for the cohort in four follow-ups (in 1983, 1986, 1995, and 2007) with a very high attrition rate (at least 94.3\% of those still alive) on all occasions [11]. In this study, the follow-up questionnaires of the cohort in 1995 and 2007 were combined with unemployment information from the longitudinal integration database for health insurance and labour market studies (acronym LISA) at Statistics Sweden. The Regional Ethical Board in Umeå, Sweden has approved the Northern Swedish cohort study. Further information about the cohort is available elsewhere [11].

\section{Definition of health}

In our study, self-rated health from the 2007 questionnaire was used as the outcome variable. Responses "fairly good" and "poor" were defined as poor and "good" was defined as good. The same question in the 1995 questionnaire was used identically to define previous health.

\section{Definitions of exposures}

A labour market status variable was created in three different ways, referred to in this study as modes of unemployment, i) self-reported long-term unemployment, ii) register-based long-term unemployment, and iii) self-reported current unemployment. For the first, at least half a year of self-reported unemployment during last three years (1993-1995) was required. For the second, at least 6 months' unemployment during 1992-1994 as reported in the LISA register database was required. In LISA, the information is limited to accumulated number of days of unemployment per year, which explains the choice of years as reported unemployment days in 1995 might have appeared after the participants' response. The first two modes respond to the research question "What is the association between high exposure to unemployment in 1995 and health in 2007?", while the last corresponds to the research question "What is the association between current unemployment 1995 and health in 2007?".

Current unemployment required a tick at unemployed for the question "What is your current employment situation?" For all three modes, employed was defined as being active in the labour market, without unemployment, which we defined as either having "Full-time employment", "Part-time (20-39 hours a week) employment" or "Labour market measure", for at least $1 \frac{1}{2}$ years, during the last three years. Response alternatives not considered as being in the labour market were "University/high-school”, “Other education”, “Casual job (<20 hours a week)”, "Sick leave”, “On parental leave”, and “Other”.

For all unemployment measures, analyses were conducted with unemployment episodes being allowed or not during the follow-up period. With the censoring, at least $1 \frac{1}{2}$ years in the labour market and no unemployment during follow-up was needed for participants to be part of analyses. Such censoring might not give an unbiased estimate of the long-term effect of unemployment, as it will not include participants who increase their future unemployment due to the unemployment before 1995, while no censoring might lead to a bias due to their exposure to unemployment during the follow-up playing an important role in future health. We consider both our alternatives to be proxies for our research questions.

\section{Confounding variables}

For all potentially confounding variables, the responses to the 1995 questionnaire, when respondents were around 30 years of age, were used. As potential confounders, we chose the variables that had been most frequently used in similar studies according to a review in 2014 of manuscripts studying selfassessed health and unemployment [1]. Our approach can help to understand what variables can be urged to be used in similar research questions without risking non-negligible biases.

Body mass index (BMI) was derived from self-reported weight and height, and calculated as weight/length ${ }^{2}$; those with a $\mathrm{BMI} \geq 30 \mathrm{~kg} / \mathrm{cm}^{2}$ were defined as obese and those with a BMI between 25 and $30 \mathrm{~kg} / \mathrm{cm}^{2}$ as overweight, and used as exposed groups. Education was divided into three groups: at most secondary education, upper secondary school and university degree, with university studies used as reference group. Based on the nomenclature used by Statistics Sweden [13], occupation was defined with low-medium white-collar work as reference group, and high white-collar and blue-collar workers as exposure groups. For marital status, married (including "living with cohabitant/partner") was used as reference group and single as exposed group. For cash margin, availability of 13,000 SEK (corresponding to 1276 euro on 24th November 2021) within a week was used as reference group and compared with no availability. For smoking, "not a current smoker" was used as reference group, and compared with "smoking at most 10 cigarettes a day", and "smoking more than 10 cigarettes a day". For social support, the instruments availability of social integration (AVSI) and availability of attachment (AVAT) were used [14]. The cut-offs for their reference groups were 13 or lower for AVSI and 10 or higher for AVAT. An index was calculated for alcohol intake based on six questions, a cut-off below 140, corresponding to low alcohol intake, was used as reference value. More details about the variables are available in a previous publication [9].

\section{Statistics}

We restricted ourselves to participants in the 1995 labour market, defined as being employed or unemployed, with complete data for all study variables. This meant that we were able to include $55-74 \%$ of the originally invited pupils in our analyses. For the analyses, estimates based on propensity scores, G- 
computation and logistic regression were used.

A risk difference was estimated based on propensity scores, using counterfactual arguments [15], with the weighting estimators suggested by Lunceford and Davidian [16]. The propensity score is the conditional probability of being assigned to the exposure group based on baseline covariates. We estimated the propensity scores with logistic regression. The counterfactual outcome for the difference estimators estimate $E[Y(1)]-E[Y(0)]$, where $E[Y(1)]$ corresponds to the expected effect if all individuals are unemployed, and $\mathrm{E}[\mathrm{Y}(0)]$ corresponds to the expected effect if all individuals are employed. Thus, the risk difference corresponds to the marginal effect of becoming unemployed.

Of the estimators, the first is the straightforward inverse probability weighting (IPW) estimator, the second is usually referred to as the augmented estimator (AUG), and the third is a doubly-robust estimator (DR). The doubly-robust estimator does not only include a weighting on the propensity score, but also makes use of the regression model from which the propensity scores are derived.

The estimators were:

$$
\begin{aligned}
& R D_{I P W}=\frac{1}{n}\left[\sum_{i=1}^{n}\left(\frac{Y_{i} X_{i}}{P S_{i}}-\frac{Y_{i}\left(1-X_{i}\right)}{1-P S_{i}}\right)\right] \\
& R D_{A U G}=\left(\sum_{i=1}^{n} \frac{X_{i}}{P S_{i}}\right)^{-1} \sum_{i=1}^{n} \frac{Y_{i} X_{i}}{P S_{i}}-\left(\sum_{i=1}^{n} \frac{1-X_{i}}{1-P S_{i}}\right)^{-1} \sum_{i=1}^{n} \frac{Y\left(1-X_{i}\right)}{1-P S_{i}} \text { and } \\
& R D_{D R}=\sum_{i=1}^{n}\left(\frac{Y_{i} X_{i}-\left(X_{i}-P S_{i}\right) m_{1}\left(X_{i}\right)}{P S_{i}}-\frac{Y_{i}\left(1-X_{i}\right)+\left(X_{i}-P S_{i}\right) m_{0}\left(X_{i}\right)}{1-P S_{i}}\right),
\end{aligned}
$$

where $Y$ refers to the outcome (self-rated health), $X$ to the exposure (employed/unemployed), $P S$ to the estimates of the propensity score. $m_{0}$ and $m_{1}$ refer to the estimated predictive value for the logistic regression used to derive propensity scores when only those defined as employed $\left(m_{0}\right)$ respectively unemployed $\left(m_{1}\right)$ are used to estimate beta coefficients.

For G-computation, logistic regression was first performed with all variables in the statistical model, including labour market status. The counterfactual outcome for the risk difference was calculated as the difference in effect if all individuals were unemployed with all individuals being employed. The Bootstrap technique with replacement was used to derive 10,000 replicates for the IPW and G-computing estimators and to calculate the mean square error (MSE) [17]. The $2.5 \%$ and $97.5 \%$ percentiles were used to calculate $95 \%$ confidence intervals. For the doubly-robust estimator, some Bootstrap replicates had too few unemployed, which caused singularity problems. Fewer replicates were then used.

The procedure for our analyses was to use all variables with logistic regression first in a "full" model, and thereafter a "reduced" model was applied with the significant variables in the full model for the self-reported long-term unemployment labour market mode. For the self-reported long-term unemployment mode, for both censored and non-censored analyses, we added or removed each potentially confounding variable individually. These results illustrate the influence of collinear variables on the effect estimates for the main exposure and for the logistic regression analyses for all variables.

R Studio was used for all statistical analyses, and its GLM procedure, with confidence intervals estimated using the profile likelihood estimator, was used for logistic regression estimates [18]. Statistical significance was defined at the $5 \%$ level.

\section{Results}

\section{General characteristics}

As only 22 participants who were unemployed in 1995 had experienced no unemployment during follow-up, the current unemployment mode with censoring could not provide reliable estimates and results are therefore not presented. For the unemployment modes that we used, the unemployment rate ranged from 8-27\% (Table 1). For other variables, only minor differences in the distribution of employed between the unemployment modes were observed. 
Table 1

Characteristics for the study populations

\section{Unemployment mode}

\section{Self-reported long-term unemployment Register-based long-term unemployment \\ Current}

unemployment

\begin{tabular}{|c|c|c|c|c|}
\hline No censor ${ }^{a}$ & Censored $^{a}$ & No censor & Censored & No censor \\
\hline
\end{tabular}

\section{Labor market status}

Employed

Unemployed

$627 \quad 78 \%$

$522 \quad 84 \%$

$178 \quad 22 \%$

585

$73 \%$

488

122

\begin{tabular}{lll}
$80 \%$ & 693 & $92 \%$ \\
\hline $20 \%$ & 61 & $8 \%$
\end{tabular}

Self-rated health 2007

Poor

$286 \quad 36 \%$

202

$\begin{array}{ll}33 \% & 283 \\ 67 \% & 516\end{array}$

\begin{tabular}{lllll}
$35 \%$ & 197 & $32 \%$ & 262 & $37 \%$ \\
\hline $65 \%$ & 413 & $68 \%$ & 492 & $63 \%$
\end{tabular}

Self-rated health 1995

Poor

$186 \quad 23$

Good

$619 \quad 77 \%$

125

$20 \%$

179

$22 \%$

\begin{tabular}{llll}
118 & $19 \%$ & 164 & $22 \%$ \\
\hline 492 & $81 \%$ & 590 & $78 \%$
\end{tabular}

Education level $^{\text {b }}$

Secondary education
Upper secondary education

University

\begin{tabular}{|l|l|}
\hline 309 & $38 \%$ \\
\hline 146 & $18 \%$ \\
\hline 350 & $43 \%$ \\
\hline
\end{tabular}

253
112
255

\begin{tabular}{l}
$41 \%$ \\
\hline $18 \%$ \\
\hline $41 \%$ \\
\hline
\end{tabular}

309
144
346

\begin{tabular}{l}
$39 \%$ \\
\hline $18 \%$ \\
\hline $43 \%$ \\
\hline
\end{tabular}

\begin{tabular}{|llll}
\hline 251 & $41 \%$ & 292 & $39 \%$ \\
\hline 110 & $18 \%$ & 134 & $18 \%$ \\
\hline 249 & $41 \%$ & 328 & $44 \%$ \\
\hline
\end{tabular}

\section{Marital status}

Married

$\begin{array}{ll}591 & 73 \% \\ 214 & 27 \%\end{array}$

$73 \%$

468

$75 \%$

581

\section{Occupation}

Blue-collar

\begin{tabular}{llllllllll}
340 & $42 \%$ & 246 & $40 \%$ & 334 & $42 \%$ & 239 & $39 \%$ & 318 & $42 \%$ \\
\hline 138 & $17 \%$ & 103 & $17 \%$ & 140 & $18 \%$ & 104 & $17 \%$ & 124 & $16 \%$ \\
\hline 327 & $41 \%$ & 271 & $44 \%$ & 325 & $41 \%$ & 267 & $44 \%$ & 312 & $41 \%$ \\
\hline
\end{tabular}

\section{Gender}

Man

Woman

\begin{tabular}{|l|l|}
376 & $47 \%$ \\
\hline 429 & $53 \%$
\end{tabular}

283

$45 \%$

367
432

\begin{tabular}{l}
$46 \%$ \\
$54 \%$ \\
\hline
\end{tabular}

278

\begin{tabular}{lll}
\hline $46 \%$ & 331 & $44 \%$ \\
\hline $54 \%$ & 423 & $56 \%$ \\
\hline
\end{tabular}

Availability of Social Integration (AVSI)

Low

High

\begin{tabular}{ll}
269 & $37 \%$ \\
\hline 509 & $73 \%$
\end{tabular}

203

$33 \%$

291
508

$36 \%$

197
413

$32 \%$

$272 \quad 36 \%$

Availability of Attachment (AVAT)

Low

High

\begin{tabular}{|l|l|}
\hline 434 & $53 \%$ \\
\hline 371 & $47 \%$ \\
\hline
\end{tabular}

321

\begin{tabular}{ll}
$52 \%$ & 433 \\
\hline $48 \%$ & 366
\end{tabular}

$54 \%$

\begin{tabular}{llll}
315 & $52 \%$ & 415 & $55 \%$ \\
\hline 295 & $48 \%$ & 339 & $45 \%$
\end{tabular}

\section{Cash margin}

Access

No access

\begin{tabular}{ll}
640 & $80 \%$ \\
\hline 165 & $20 \%$
\end{tabular}

514

$83 \%$

639

$80 \%$

\begin{tabular}{llll}
508 & $83 \%$ & 605 & $80 \%$ \\
\hline 102 & $17 \%$ & 149 & $20 \%$ \\
\hline
\end{tabular}

\section{Smoking}

Not smoking

$561 \quad 70 \%$

449

$72 \%$

558

$70 \%$

444

$73 \% \quad 528 \quad 70 \%$

a Censored individuals were during the follow-up period between autumn 1995 and autumn 2007 either unemployed or were active in the labor market for too short period.

${ }^{b}$ Secondary education corresponds to at most 2-years of secondary education, and upper-secondary education corresponds to 3-4 years of secondary education 


\begin{tabular}{|c|c|c|c|c|c|c|c|c|c|c|}
\hline \multirow[b]{3}{*}{ Smoking $\leq 10$ cigarettes } & \multicolumn{10}{|c|}{ Unemployment mode } \\
\hline & \multicolumn{4}{|c|}{ Self-reported long-term unemployment } & \multicolumn{4}{|c|}{ Register-based long-term unemployment } & \multicolumn{2}{|c|}{$\begin{array}{l}\text { Current } \\
\text { unemployment }\end{array}$} \\
\hline & 152 & $19 \%$ & 113 & $18 \%$ & 147 & $18 \%$ & 108 & $18 \%$ & 139 & $18 \%$ \\
\hline Smoking $>10$ cigarettes & 92 & $11 \%$ & 58 & $9 \%$ & 94 & $12 \%$ & 58 & $10 \%$ & 87 & $12 \%$ \\
\hline \multicolumn{11}{|l|}{ Alcohol intake } \\
\hline Low & 399 & $50 \%$ & 313 & $50 \%$ & 389 & $49 \%$ & 306 & $50 \%$ & 360 & $48 \%$ \\
\hline High & 406 & $50 \%$ & 307 & $50 \%$ & 410 & $51 \%$ & 304 & $50 \%$ & 394 & $52 \%$ \\
\hline \multicolumn{11}{|l|}{ Body mass index } \\
\hline Normal & 511 & $63 \%$ & 393 & $63 \%$ & 508 & $64 \%$ & 387 & $63 \%$ & 474 & $63 \%$ \\
\hline Overweight & 247 & $31 \%$ & 191 & $31 \%$ & 245 & $31 \%$ & 188 & $31 \%$ & 234 & $31 \%$ \\
\hline Obese & 47 & $6 \%$ & 36 & $6 \%$ & 46 & $6 \%$ & 35 & $6 \%$ & 46 & $6 \%$ \\
\hline \multicolumn{11}{|c|}{$\begin{array}{l}\text { a Censored individuals were during the follow-up period between autumn } 1995 \text { and autumn } 2007 \text { either unemployed or were active in the labor market fo } \\
\text { too short period. }\end{array}$} \\
\hline
\end{tabular}

\section{Estimates of the long-term effect of unemployment}

For the unemployment modes, most estimates showed a statistically significant negative long-term effect of unemployment when it was compared with being employed (Table 2). The statistical models with all potential confounders had a higher point estimate than the models with only significant confounders, the only exceptions being the logistic regression and the G-computation estimator for the register-based unemployment mode with censoring. Thus, adding more variables mainly increased the estimated effect of unemployment. Applying censoring for unemployment episodes lowered the negative effect of unemployment for all analyses compared to not censoring for unemployment during follow-up, ranging from an absolute risk difference of 0.001 to 0.039 between these estimates. 
Table 2

Long-term effect on self-rated health from unemployment at 40 years of age for different estimators and unemployment mode.

\begin{tabular}{|c|c|c|c|c|c|c|c|c|}
\hline \multirow{2}{*}{$\begin{array}{l}\text { Unemployment } \\
\text { mode }\end{array}$} & \multirow{2}{*}{$\begin{array}{l}\text { Unemployment } \\
\text { during } \\
\text { follow-up }\end{array}$} & \multicolumn{3}{|c|}{ Estimator ${ }^{a}$} & \multirow[b]{2}{*}{$\begin{array}{l}\text { IPW } \\
\text { standard }\end{array}$} & \multirow[b]{2}{*}{$\begin{array}{l}\text { IPW } \\
\text { augmented }\end{array}$} & \multirow[b]{2}{*}{$\begin{array}{l}\text { IPW } \\
\text { doubly } \\
\text { robust }\end{array}$} & \multirow[b]{2}{*}{$\begin{array}{l}\text { MSEc, } \\
\text { low/high }\end{array}$} \\
\hline & & $\begin{array}{l}\text { Employed/ } \\
\text { unemployed }\end{array}$ & $\begin{array}{l}\text { Logistic } \\
\text { regression }\end{array}$ & $\begin{array}{l}\text { G- } \\
\text { computation }\end{array}$ & & & & \\
\hline \multicolumn{9}{|l|}{ All confounders ${ }^{d}$} \\
\hline \multirow{4}{*}{$\begin{array}{l}\text { Self-reported long-term } \\
\text { unemployment }\end{array}$} & \multirow[t]{2}{*}{ No censor } & \multirow[t]{2}{*}{$627 / 178$} & 1.90 & 0.134 & 0.129 & 0.128 & 0.125 & \multirow{2}{*}{$\begin{array}{l}0.0018 / \\
0.0019\end{array}$} \\
\hline & & & $(1.31-2.76)$ & $(0.051-0.22)$ & $\begin{array}{l}(0.045- \\
0.21)\end{array}$ & $\begin{array}{l}(0.042- \\
0.21)\end{array}$ & $\begin{array}{l}(0.041- \\
0.21)\end{array}$ & \\
\hline & \multirow[t]{2}{*}{ Censor } & \multirow[t]{2}{*}{$522 / 98$} & 1.73 & 0.111 & 0.114 & 0.108 & 0.124 & \multirow{2}{*}{$\begin{array}{l}0.0028 / \\
0.0038\end{array}$} \\
\hline & & & $(1.07-2.81)$ & $(0.008-0.22)$ & $\begin{array}{l}(0.001- \\
0.25)\end{array}$ & $\begin{array}{l}(-0.005- \\
0.23)\end{array}$ & $\begin{array}{l}(0.022- \\
0.23)\end{array}$ & \\
\hline \multirow[t]{4}{*}{$\begin{array}{l}\text { Register-based long-term } \\
\text { unemployment }\end{array}$} & \multirow[t]{2}{*}{ No censor } & \multirow[t]{2}{*}{$585 / 214$} & 1.65 & 0.103 & 0.104 & 0.102 & 0.099 & \multirow[t]{2}{*}{$\begin{array}{l}0.0014 / \\
0.0015\end{array}$} \\
\hline & & & $(1.16-2.34)$ & $(0.027-0.18)$ & $\begin{array}{l}(0.028- \\
0.18)\end{array}$ & $\begin{array}{l}(0.025- \\
0.18)\end{array}$ & $\begin{array}{l}(0.025- \\
0.17)\end{array}$ & \\
\hline & \multirow[t]{2}{*}{ Censor } & \multirow[t]{2}{*}{$488 / 122$} & 1.38 & 0.064 & 0.067 & 0.069 & 0.074 & \multirow{2}{*}{$\begin{array}{l}0.0022 / \\
0.0023\end{array}$} \\
\hline & & & $(0.88-2.17)$ & $(-0.027-0.16)$ & $\begin{array}{l}(-0.025- \\
0.17)\end{array}$ & $\begin{array}{l}(-0.025- \\
0.16)\end{array}$ & $\begin{array}{l}(-0.015- \\
0.17)\end{array}$ & \\
\hline \multirow[t]{2}{*}{ Current unemployment } & \multirow[t]{2}{*}{ No censor } & \multirow[t]{2}{*}{$693 / 61$} & 1.76 & 0.121 & 0.199 & 0.253 & 0.252 & $0.0052 /$ \\
\hline & & & $(0.97-3.18)$ & $(-0.018-0.26)$ & $\begin{array}{l}(0.006- \\
0.48)\end{array}$ & $\begin{array}{l}(0.057- \\
0.44)\end{array}$ & $\begin{array}{l}(-0.003- \\
0.43)\end{array}$ & 0.0147 \\
\hline \multicolumn{9}{|l|}{ Significant confounders ${ }^{e}$} \\
\hline \multicolumn{9}{|c|}{$\begin{array}{l}\text { a Confidence intervals, corresponding to the } 2.5 \% \text { and } 97.5 \text { Bootstrap percentiles, are presented within parentheses. Estimates represent the health effect } \\
\text { on unemployed compared to employed individuals as represented with relative risk for logistic regression (above } 0 \text { a poorer health) and risk difference } \\
\text { (above } 1 \text { a poorer health) for other estimators. }\end{array}$} \\
\hline \multicolumn{9}{|c|}{ b Participants were censored or not censored if they experienced unemployment between 1995 and 2007.} \\
\hline \multicolumn{9}{|c|}{${ }^{\mathrm{c}}$ MSE = Mean squared error. The lowest and highest MSE for the estimators of the definition is presented. } \\
\hline \multicolumn{9}{|c|}{$\begin{array}{l}\text { d Analyzes are controlling for alcohol intake, Availability of Attachment (AVAT), Availability of Social Integration (AVSI), body mass index, cash margin, } \\
\text { education level, gender, marital status, previous health status, occupation and smoking. }\end{array}$} \\
\hline \multicolumn{9}{|c|}{$\begin{array}{l}\text { e Significant confounders were determined with logistic regression. Analyzes are controlling for education level, marital status, previous health status and } \\
\text { occupation. }\end{array}$} \\
\hline \multicolumn{9}{|c|}{ IPW = inverse probability weighting estimator; Risk differences are used for the G-computation and IPW estimators. } \\
\hline
\end{tabular}




\begin{tabular}{|c|c|c|c|c|c|c|c|c|}
\hline \multirow{2}{*}{$\begin{array}{l}\text { Unemployment } \\
\text { mode }\end{array}$} & \multirow{2}{*}{$\begin{array}{l}\text { Unemployment } \\
\text { during } \\
\text { follow-up }\end{array}$} & \multicolumn{3}{|c|}{ Estimator $^{a}$} & \multirow[b]{2}{*}{$\begin{array}{l}\text { IPW } \\
\text { standard }\end{array}$} & \multirow[b]{2}{*}{$\begin{array}{l}\text { IPW } \\
\text { augmented }\end{array}$} & \multirow[b]{2}{*}{$\begin{array}{l}\text { IPW } \\
\text { doubly } \\
\text { robust }\end{array}$} & \multirow[b]{2}{*}{$\begin{array}{l}\text { MSE } \\
\text { low/high }\end{array}$} \\
\hline & & $\begin{array}{l}\text { Employed/, } \\
\text { unemployed }\end{array}$ & $\begin{array}{l}\text { Logistic } \\
\text { regression }\end{array}$ & $\begin{array}{l}\text { G- } \\
\text { computation }\end{array}$ & & & & \\
\hline \multirow{4}{*}{$\begin{array}{l}\text { Self-reported long-term } \\
\text { unemployment }\end{array}$} & \multirow[t]{2}{*}{ No censor } & \multirow[t]{2}{*}{$627 / 178$} & 1.85 & 0.129 & 0.123 & 0.123 & 0.120 & \multirow{2}{*}{$\begin{array}{l}0.0018 / \\
0.0021\end{array}$} \\
\hline & & & $(1.28-2.67)$ & $(0.049-0.21)$ & $\begin{array}{l}(0.042- \\
0.20)\end{array}$ & $\begin{array}{l}(0.041- \\
0.20)\end{array}$ & $\begin{array}{l}(0.040- \\
0.20)\end{array}$ & \\
\hline & \multirow[t]{2}{*}{ Censor } & \multirow[t]{2}{*}{522 / 98} & 1.74 & 0.113 & 0.103 & 0.103 & 0.101 & \multirow{2}{*}{$\begin{array}{l}0.0025 / \\
0.0027\end{array}$} \\
\hline & & & $(1.08-2.79)$ & $(0.013-0.22)$ & $\begin{array}{l}(0.0005- \\
0.21)\end{array}$ & $\begin{array}{l}(0.0002- \\
0.21)\end{array}$ & $\begin{array}{l}(0.004- \\
0.20)\end{array}$ & \\
\hline \multirow{4}{*}{$\begin{array}{l}\text { Register-based long-term } \\
\text { unemployment }\end{array}$} & \multirow[t]{2}{*}{ No censor } & \multirow[t]{2}{*}{$585 / 214$} & 1.63 & 0.102 & 0.096 & 0.097 & 0.095 & \multirow{2}{*}{$\begin{array}{l}0.0014 / \\
0.0014\end{array}$} \\
\hline & & & $(1.16-2.31)$ & $(0.028-0.17)$ & $\begin{array}{l}(0.022- \\
0.17)\end{array}$ & $\begin{array}{l}(0.022- \\
0.17)\end{array}$ & $\begin{array}{l}(0.021- \\
0.17)\end{array}$ & \\
\hline & \multirow[t]{2}{*}{ Censor } & \multirow[t]{2}{*}{488 / 122} & 1.40 & 0.066 & 0.059 & 0.059 & 0.057 & \multirow{2}{*}{$\begin{array}{l}0.0020 / \\
0.0021\end{array}$} \\
\hline & & & $(0.89-2.16)$ & $(-0.022-0.16)$ & $\begin{array}{l}(-0.029- \\
0.15)\end{array}$ & $\begin{array}{l}(-0.029- \\
0.15)\end{array}$ & $\begin{array}{l}(-0.028- \\
0.15)\end{array}$ & \\
\hline \multirow[t]{2}{*}{ Current unemployment } & \multirow[t]{2}{*}{ No censor } & \multirow[t]{2}{*}{$693 / 61$} & 1.66 & 0.109 & 0.156 & 0.155 & 0.163 & \multirow{2}{*}{$\begin{array}{l}0.0046 \\
/ 0.0072\end{array}$} \\
\hline & & & $(0.94-2.91)$ & $(-0.019-0.25)$ & $\begin{array}{l}(-0.003- \\
0.33)\end{array}$ & $\begin{array}{l}(-0.005- \\
0.32)\end{array}$ & $\begin{array}{l}(0.012- \\
0.32)\end{array}$ & \\
\hline \multicolumn{9}{|c|}{$\begin{array}{l}\text { a Confidence intervals, corresponding to the } 2.5 \% \text { and } 97.5 \text { Bootstrap percentiles, are presented within parentheses. Estimates represent the health effect } \\
\text { on unemployed compared to employed individuals as represented with relative risk for logistic regression (above } 0 \text { a poorer health) and risk difference } \\
\text { (above } 1 \text { a poorer health) for other estimators. }\end{array}$} \\
\hline \multicolumn{9}{|c|}{ b Participants were censored or not censored if they experienced unemployment between 1995 and 2007.} \\
\hline \multicolumn{9}{|c|}{${ }^{\mathrm{c}} \mathrm{MSE}=$ Mean squared error. The lowest and highest MSE for the estimators of the definition is presented. } \\
\hline \multicolumn{9}{|c|}{$\begin{array}{l}\text { d Analyzes are controlling for alcohol intake, Availability of Attachment (AVAT), Availability of Social Integration (AVSI), body mass index, cash margin, } \\
\text { education level, gender, marital status, previous health status, occupation and smoking. }\end{array}$} \\
\hline \multicolumn{9}{|c|}{$\begin{array}{l}\text { e Significant confounders were determined with logistic regression. Analyzes are controlling for education level, marital status, previous health status and } \\
\text { occupation. }\end{array}$} \\
\hline \multicolumn{9}{|c|}{ IPW = inverse probability weighting estimator; Risk differences are used for the G-computation and IPW estimators. } \\
\hline
\end{tabular}

For most unemployment modes, there was a rather small difference in effect estimates for the IPW estimators. The G-estimator gave a much smaller effect estimate for current unemployment mode than the IPW estimators while the difference was smaller for the other analyses, although in general greater than the one between the IPW estimators.

There were notable differences between estimates for the different unemployment definitions. The smallest effects were observed for the register-based longterm unemployment definition where the risk difference estimators ranged between $0.06-0.07$ with censoring, while two IPW estimates of the current unemployment definition showed an effect as large as 0.25 poorer health for unemployed. For logistic regression, the estimates varied considerably with the odds ratio ranging from 1.4 to 1.9 , with six of ten estimates giving statistical significance.

\section{The influence of confounders on effect estimates}

In Table 3 and 4, results are shown based on variables included in the analysis. In them, model 1 presents the result when all potentially confounding variables are included in the analysis and model 13 presents a reduced model where only variables significant in the logistic regression analyses are included.

Consequently, model 16 for instance will present the result when all significant variables are used except for previous health. 
Table 3

Logistic regression estimates for self-reported long-term unemployment, with or without censoring for unemployment during follow-up.

\begin{tabular}{|c|c|c|c|c|c|c|c|c|c|c|c|c|c|c|c|}
\hline \multirow[b]{2}{*}{ Model } & \multirow{2}{*}{$\begin{array}{l}\begin{array}{l}\text { Labor } \\
\text { market } \\
\text { status }\end{array} \\
\text { UE }\end{array}$} & \multicolumn{2}{|c|}{$\begin{array}{l}\text { Education } \\
\text { level }\end{array}$} & \multirow{2}{*}{$\begin{array}{l}\begin{array}{l}\text { Marital } \\
\text { status }\end{array} \\
\text { Single }\end{array}$} & \multirow{2}{*}{$\begin{array}{l}\begin{array}{l}\text { Prev. } \\
\text { health }\end{array} \\
\text { Poor }\end{array}$} & \multicolumn{2}{|c|}{ Occupation } & \multirow{2}{*}{$\begin{array}{l}\text { Sex } \\
\text { Male }\end{array}$} & \multicolumn{2}{|c|}{$\begin{array}{l}\text { Social } \\
\text { network }\end{array}$} & \multicolumn{2}{|c|}{ Cash margin } & \multirow{2}{*}{$\begin{array}{r}\text { Smoking } \\
\quad>10\end{array}$} & \multicolumn{2}{|c|}{ Alcohol } \\
\hline & & Med & High & & & Med & High & & $\begin{array}{l}\text { AVAT } \\
\text { high }\end{array}$ & $\begin{array}{l}\text { AVSI } \\
\text { low }\end{array}$ & $\begin{array}{l}\text { No } \\
\text { access }\end{array}$ & $\leq 10$ & & High & Over-weigh \\
\hline
\end{tabular}

No

censoring

$(n=805)^{a}$

\begin{tabular}{|c|c|c|c|c|c|c|c|c|c|c|c|c|c|c|c|}
\hline Model 1 & $1.90 *$ & $0.57^{\star}$ & $0.60 *$ & $1.65^{\star}$ & $4.03^{\star}$ & 0.75 & $0.54^{*}$ & 1.11 & 0.97 & 1.10 & 0.95 & 1.30 & 1.20 & 0.74 & 1.07 \\
\hline Model 2 & $1.83^{\star}$ & - & - & $1.67 *$ & $3.93^{\star}$ & 0.77 & 0.71 & 1.03 & 0.99 & 1.12 & 0.95 & 1.29 & 1.17 & 0.71 & 1.05 \\
\hline Model 3 & $1.92^{\star}$ & $0.56^{*}$ & $0.59 *$ & - & $4.03^{\star}$ & 0.75 & $0.54^{\star}$ & 1.18 & 0.96 & 1.10 & 1.02 & 1.32 & 1.26 & 0.78 & 1.05 \\
\hline Model 4 & $1.93^{\star}$ & $0.59 *$ & 0.66 & $1.65^{\star}$ & - & 0.79 & $0.55^{\star}$ & 1.08 & 0.90 & 1.02 & 1.15 & 1.51 * & 1.59 & 0.77 & 1.11 \\
\hline Model 5 & $1.91^{\star}$ & 0.65 & 0.84 & $1.66^{*}$ & $4.00 *$ & - & - & 1.13 & 0.98 & 1.10 & 1.08 & 1.34 & 1.28 & 0.71 & 1.06 \\
\hline Model 6 & $1.89 *$ & $0.58^{*}$ & $0.61 *$ & $1.67^{*}$ & $4.02^{\star}$ & 0.73 & $0.54^{*}$ & - & 0.97 & 1.11 & 0.93 & 1.28 & 1.18 & 0.76 & 1.08 \\
\hline Model 7 & $1.90 *$ & $0.57 *$ & 0.60 * & $1.65^{\star}$ & $4.04^{\star}$ & 0.75 & $0.54^{\star}$ & 1.11 & - & 1.10 & 0.95 & 1.30 & 1.20 & 0.74 & 1.07 \\
\hline Model 8 & $1.88^{*}$ & $0.57 *$ & $0.59 *$ & $1.65^{\star}$ & $4.00^{\star}$ & 0.74 & $0.54^{\star}$ & 1.11 & 0.99 & - & 0.94 & 1.30 & 1.21 & 0.74 & 1.07 \\
\hline Model 9 & $1.89 *$ & $0.57 *$ & $0.60 *$ & $1.64^{\star}$ & $4.01^{*}$ & 0.75 & $0.55^{\star}$ & 1.11 & 0.97 & 1.11 & - & 1.30 & 1.20 & 0.74 & 1.07 \\
\hline Model 10 & $1.93^{*}$ & $0.56^{*}$ & $0.61^{*}$ & $1.67 *$ & $4.16^{\star}$ & 0.74 & $0.53^{*}$ & 1.06 & 0.97 & 1.10 & 0.95 & - & - & 0.77 & 1.07 \\
\hline Model 11 & $1.86^{\star}$ & $0.55^{\star}$ & $0.58^{\star}$ & $1.59 *$ & $3.99 *$ & 0.74 & $0.53^{*}$ & 0.99 & 0.98 & 1.09 & 0.95 & 1.23 & 1.15 & - & 1.06 \\
\hline Model 12 & $1.91^{*}$ & $0.58^{*}$ & $0.61^{*}$ & $1.68 *$ & $4.12^{\star}$ & 0.74 & $0.55^{\star}$ & 1.13 & 0.96 & 1.11 & 0.94 & 1.29 & 1.20 & 0.73 & - \\
\hline Model 13 & $1.85^{\star}$ & $0.57 *$ & $0.54^{*}$ & $1.85^{\star}$ & $4.13^{\star}$ & 0.73 & $0.54^{*}$ & - & - & - & - & - & - & - & - \\
\hline Model 14 & $1.77^{*}$ & - & - & $1.61^{*}$ & $4.00 *$ & 0.77 & $0.70 *$ & - & - & - & - & - & - & - & - \\
\hline Model 15 & $1.89 *$ & $0.58^{\star}$ & $0.61^{*}$ & - & $4.20^{*}$ & 0.72 & $0.53^{*}$ & - & - & - & - & - & - & - & - \\
\hline Model 16 & $2.00 *$ & $0.59 *$ & 0.70 & $1.69 *$ & - & 0.76 & $0.51 *$ & - & - & - & - & - & - & - & - \\
\hline Model 17 & $1.90^{*}$ & 0.65 & 0.87 & 1.65 & $4.20^{\star}$ & - & - & - & - & - & - & - & - & - & - \\
\hline Model 18 & $1.85^{\star}$ & $0.57 *$ & $0.60 *$ & $1.62^{\star}$ & $4.14^{\star}$ & 0.73 & $0.54^{*}$ & 1.01 & - & - & - & - & - & - & - \\
\hline Model 19 & $1.85^{\star}$ & $0.57 *$ & $0.60 *$ & $1.62^{*}$ & $4.13^{\star}$ & 0.73 & $0.54^{*}$ & - & 0.98 & - & - & - & - & - & - \\
\hline Model 20 & $1.87^{\star}$ & $0.57 *$ & $0.61^{*}$ & $1.63^{*}$ & $4.17^{\star}$ & 0.74 & $0.53^{*}$ & - & - & 1.09 & - & - & - & - & - \\
\hline Model 21 & $1.87^{\star}$ & $0.57 *$ & $0.60 *$ & $1.63^{*}$ & $4.17^{\star}$ & 0.72 & $0.53^{*}$ & - & - & - & 0.94 & - & - & - & - \\
\hline Model 22 & $1.83^{*}$ & $0.57 *$ & $0.60 *$ & $1.61^{*}$ & $4.02^{\star}$ & 0.73 & $0.55^{\star}$ & - & - & - & - & 1.21 & 1.15 & - & - \\
\hline Model 23 & $1.88^{*}$ & $0.59 *$ & $0.63^{*}$ & $1.70^{*}$ & $4.17^{\star}$ & 0.73 & $0.55^{\star}$ & - & - & - & - & - & - & 0.79 & - \\
\hline Model 24 & $1.86^{\star}$ & $0.55^{\star}$ & $0.59 *$ & $1.60 *$ & $4.05^{\star}$ & 0.74 & $0.53^{*}$ & - & - & - & - & - & - & - & 1.07 \\
\hline \multirow[t]{2}{*}{ Crude } & $2.06 *$ & 0.79 & 1.12 & $1.76^{*}$ & $4.38 *$ & 0.77 & $0.57 *$ & 0.95 & 0.86 & 0.87 & 1.59 & $1.61 *$ & $1.89 *$ & 0.89 & 1.10 \\
\hline & $\begin{array}{l}(1.5- \\
2.9)\end{array}$ & & & & & & & & & & & & & & \\
\hline
\end{tabular}

\section{Censoring}

$(n=620)^{a}$

Model 1

$1.73^{\star}$

0.60

0.50 * $1.77 *$

$4.54^{*}$

0.80

$0.49 *$

1.10

1.05

$1.14 \quad 0.92$

1.50

$\begin{array}{lll}0.85 & 0.79 & 1.11\end{array}$

a Participants were censored (excluded) or not censored (included) if they experience unemployment between 1995 and 2007.

UE=unemployed. Prev. health = previous health as measured in 1995. Estimates represent the health effect on unemployed (exposed) compared to employed exposed) individuals.

Models: Model 1 - controlling for alcohol intake, Availability of Attachment (AVAT), Availability of Social Integration (AVSI), body mass index, cash margin, er level, gender, marital status, previous health status, occupation and smoking; Model 2: model 1 - education level; Model 3: model 1 - marital status; Model 4: previous health; Model 5: model 1 - occupation; Model 6: model 1 - sex; Model 7: model 1 - AVAT; Model 8: model 1 - AVSI; Model 9: model 1 - cash margin model 1 - smoking; Model 11: model 1 - alcohol intake; Model 12: model 1 - obesity; Model 13: significant terms in full model, i.e education level, marital sta health status and occupation; Model 14: model 13 - education level; Model 15: model 13 - marital status; Model 16: model 13 - previous health; Model 17: $n$ occupation; Model 18: model 13 + sex; Model 19: model 13 + AVAT; Model 20: model 13 + AVSl; Model 21: model 13 + cash margin; Model 22: model 13 + sm Model 23: model 13 + alcohol intake; Model 24: model 13 + obesity. Note: "-" means that this variable was excluded in analyses compared to either model $1 \mathrm{c}$ means that the variable was added to the significant variables in the analyses. 


\begin{tabular}{|c|c|c|c|c|c|c|c|c|c|c|c|c|c|c|c|}
\hline \multirow[b]{2}{*}{ Model 2} & \multirow{2}{*}{$\begin{array}{l}\begin{array}{l}\text { Labor } \\
\text { market } \\
\text { status }\end{array} \\
1.66^{\star}\end{array}$} & \multicolumn{2}{|c|}{$\begin{array}{l}\text { Education } \\
\text { level }\end{array}$} & \multirow{2}{*}{$\begin{array}{c}\begin{array}{c}\text { Marital } \\
\text { status }\end{array} \\
1.77^{\star}\end{array}$} & \multirow{2}{*}{$\begin{array}{l}\text { Prev. } \\
\text { health }\end{array}$} & \multicolumn{2}{|c|}{ Occupation } & \multirow{2}{*}{$\begin{array}{l}\text { Sex } \\
\\
1.02\end{array}$} & \multicolumn{2}{|c|}{$\begin{array}{l}\text { Social } \\
\text { network }\end{array}$} & \multicolumn{2}{|c|}{ Cash margin } & \multirow{2}{*}{$\begin{array}{r}\text { Smoking } \\
0.84\end{array}$} & \multicolumn{2}{|c|}{ Alcohol } \\
\hline & & - & - & & & 0.88 & 0.73 & & 1.08 & 1.15 & 0.91 & 1.46 & & 0.75 & 1.09 \\
\hline Model 3 & $1.73^{*}$ & 0.60 & $0.51^{*}$ & - & $4.46^{\star}$ & 0.81 & $0.50 *$ & 1.16 & 1.04 & 1.15 & 1.01 & 1.50 & 0.90 & 0.83 & 1.12 \\
\hline Model 4 & $1.71^{\star}$ & 0.59 & $0.57 *$ & $1.71^{\star}$ & - & 0.88 & $0.49 *$ & 1.06 & 0.98 & 1.07 & 1.18 & 1.66 & 1.22 & 0.82 & 1.23 \\
\hline Model 5 & $1.72^{\star}$ & 0.72 & 0.77 & $1.75^{\star}$ & $4.55^{\star}$ & - & - & 1.11 & 1.07 & 1.12 & 1.01 & 1.55 & 0.95 & 0.74 & 1.10 \\
\hline Model 6 & $1.72^{\star}$ & 0.72 & $0.61^{*}$ & $1.79 *$ & $4.52^{\star}$ & 0.78 & $0.49 *$ & - & 1.04 & 1.14 & 0.90 & 1.47 & 0.84 & 0.81 & 1.13 \\
\hline Model 7 & $1.74^{\star}$ & 0.60 & $0.50 *$ & $1.77^{\star}$ & $4.53^{\star}$ & 0.80 & $0.49 *$ & 1.10 & - & 1.15 & 0.92 & 1.50 & 0.85 & 0.79 & 1.11 \\
\hline Model 8 & $1.72^{\star}$ & 0.60 & 0.50 * & $1.78^{\star}$ & $4.51^{\star}$ & 0.79 & $0.49 *$ & 1.11 & 1.08 & - & 0.91 & 1.48 & 0.85 & 0.80 & 1.12 \\
\hline Model 9 & $1.73^{\star}$ & 0.60 & $0.50 *$ & $1.76 *$ & $4.49 *$ & 0.81 & $0.49 *$ & 1.12 & 1.05 & 1.14 & - & 1.50 & 0.85 & 0.79 & 1.11 \\
\hline Model 10 & $1.76^{\star}$ & 0.59 & $0.51^{*}$ & $1.75^{\star}$ & $4.50^{\star}$ & 0.81 & $0.49 *$ & 1.07 & 1.05 & 1.10 & 0.92 & - & - & 0.82 & 1.11 \\
\hline Model 11 & $1.70 *$ & 0.58 & $0.49 *$ & $1.74^{\star}$ & $4.50^{\star}$ & 0.79 & $0.47^{\star}$ & 1.02 & 1.06 & 1.12 & 0.93 & 1.43 & 0.82 & - & 1.11 \\
\hline Model 12 & $1.77^{\star}$ & 0.62 & $0.11^{*}$ & $1.82^{\star}$ & $4.72^{\star}$ & 0.78 & $0.49 *$ & 1.14 & 1.03 & 1.14 & 0.91 & 1.49 & 0.85 & 0.78 & - \\
\hline Model 13 & $1.77^{\star}$ & $0.58^{*}$ & $0.62^{*}$ & $1.63^{\star}$ & $4.27^{\star}$ & 0.73 & $0.54^{*}$ & - & - & - & - & - & - & - & - \\
\hline Model 14 & $1.69 *$ & - & - & $1.61^{*}$ & $4.13^{*}$ & 0.77 & $0.70^{*}$ & - & - & - & - & - & - & - & - \\
\hline Model 15 & $1.82^{\star}$ & $0.59 *$ & $0.62^{*}$ & - & $4.35^{\star}$ & 0.71 & $0.53^{*}$ & - & - & - & - & - & - & - & - \\
\hline Model 16 & $1.88^{*}$ & $0.61 *$ & 0.72 & $1.71^{*}$ & - & 0.77 & $0.51 *$ & - & - & - & - & - & - & - & - \\
\hline Model 17 & $1.83^{\star}$ & 0.66 & 0.88 & $1.65^{\star}$ & $4.32^{\star}$ & - & - & - & - & - & - & - & - & - & - \\
\hline Model 18 & $1.77^{\star}$ & $0.58^{*}$ & $0.62^{*}$ & $1.62^{\star}$ & $4.27^{\star}$ & 0.73 & $0.54^{\star}$ & 1.02 & - & - & - & - & - & - & - \\
\hline Model 19 & $1.77^{\star}$ & $0.58^{*}$ & $0.62^{\star}$ & $1.63^{\star}$ & $4.27^{\star}$ & 0.73 & $0.54^{\star}$ & - & 1.00 & - & - & - & - & - & - \\
\hline Model 20 & $1.79 *$ & $0.58^{\star}$ & $0.62^{\star}$ & $1.63^{\star}$ & $4.32^{\star}$ & 0.74 & $0.54 *$ & - & - & 1.13 & - & - & - & - & - \\
\hline Model 21 & $1.77^{\star}$ & $0.58^{*}$ & $0.62^{\star}$ & $1.62^{\star}$ & $4.26^{\star}$ & 0.73 & $0.54^{\star}$ & - & - & - & 1.02 & - & - & - & - \\
\hline Model 22 & $1.74^{\star}$ & $0.59 *$ & $0.61^{*}$ & $1.60 *$ & $4.10^{\star}$ & 0.73 & $0.55^{\star}$ & - & - & - & - & 1.31 & 1.25 & - & - \\
\hline Model 23 & $1.81^{\star}$ & $0.60 *$ & $0.64^{*}$ & $1.69 *$ & $4.31^{\star}$ & 0.72 & $0.55^{\star}$ & - & - & - & - & - & - & 0.81 & - \\
\hline Model 24 & $1.77^{\star}$ & $0.56^{*}$ & 0.60 * & $1.60 *$ & $4.17^{\star}$ & 0.74 & $0.53^{\star}$ & - & - & - & - & - & - & - & 1.05 \\
\hline Crude & $\begin{array}{l}1.78 \text { * } \\
(1.1- \\
2.8)\end{array}$ & 0.80 & 1.02 & $1.74^{*}$ & $4.54^{\star}$ & 0.88 & $0.61^{*}$ & 0.94 & 1.02 & 0.97 & 1.45 & $1.69 *$ & 1.45 & 0.89 & 1.23 \\
\hline
\end{tabular}

a Participants were censored (excluded) or not censored (included) if they experience unemployment between 1995 and 2007.

UE=unemployed. Prev. health = previous health as measured in 1995. Estimates represent the health effect on unemployed (exposed) compared to employed exposed) individuals.

Models: Model 1 - controlling for alcohol intake, Availability of Attachment (AVAT), Availability of Social Integration (AVSI), body mass index, cash margin, es level, gender, marital status, previous health status, occupation and smoking; Model 2: model 1 - education level; Model 3: model 1 - marital status; Model 4: previous health; Model 5: model 1 - occupation; Model 6: model 1 - sex; Model 7: model 1 - AVAT; Model 8: model 1 - AVSI; Model 9: model 1 - cash margin model 1 - smoking; Model 11: model 1 - alcohol intake; Model 12: model 1 - obesity: Model 13: significant terms in full model, i.e education level, marital sta health status and occupation; Model 14: model 13 - education level; Model 15: model 13 - marital status; Model 16: model 13 - previous health; Model 17: m occupation; Model 18: model 13 + sex; Model 19: model 13 + AVAT; Model 20: model 13 + AVSI; Model 21: model 13 + cash margin; Model 22: model 13 + sm Model 23: model 13 + alcohol intake; Model 24: model 13 + obesity. Note: "-" means that this variable was excluded in analyses compared to either model 1 c means that the variable was added to the significant variables in the analyses. 
Table 4

Estimates of the health effect of unemployment on health for different variable setups for the logistic regression, G-computation and inverse propensity weighting (IPW) estimators for self-reported long-term unemployment.

\begin{tabular}{|c|c|c|c|c|c|}
\hline \multirow[t]{3}{*}{ Model } & \multirow{3}{*}{$\begin{array}{l}\text { Unemployment during } \\
\text { follow-up }\end{array}$} & \multicolumn{4}{|l|}{ Risk difference } \\
\hline & & G-computation & IPW standard & IPW augmented & IPW \\
\hline & & & & & doubly robust \\
\hline \multirow[t]{2}{*}{ Model $1(\text { full })^{b}$} & No censor & $0.134^{*}$ & $0.129^{\star}$ & $0.128^{*}$ & $0.125^{\star}$ \\
\hline & Censor & $0.111^{*}$ & $0.114^{\star}$ & 0.108 & $0.124^{\star}$ \\
\hline \multirow{2}{*}{$\begin{array}{l}\text { Model 2: } \\
\text { model } 1 \text { - education level }\end{array}$} & No censor & $0.126^{*}$ & $0.130 *$ & $0.130^{\star}$ & $0.129 *$ \\
\hline & Censor & $0.103^{*}$ & 0.117 & 0.115 & $0.126^{\star}$ \\
\hline \multirow{2}{*}{$\begin{array}{l}\text { Model 3: } \\
\text { model } 1 \text { - marital status }\end{array}$} & No censor & $0.138^{*}$ & $0.136^{\star}$ & $0.136^{\star}$ & $0.133^{*}$ \\
\hline & Censor & $0.111 *$ & 0.116 & 0.109 & $0.131^{*}$ \\
\hline \multirow{2}{*}{$\begin{array}{l}\text { Model 4: } \\
\text { model } 1 \text { - previous health }\end{array}$} & No censor & $0.148^{*}$ & $0.145^{\star}$ & $0.142^{*}$ & $0.143^{*}$ \\
\hline & Censor & $0.117^{*}$ & $0.119 *$ & 0.113 & $0.125^{\star}$ \\
\hline \multirow{2}{*}{$\begin{array}{l}\text { Model } 5: \\
\text { model } 1 \text { - occupation }\end{array}$} & No censor & $0.136^{*}$ & $0.127^{\star}$ & $0.128^{*}$ & $0.125^{\star}$ \\
\hline & Censor & $0.111^{*}$ & $0.116^{\star}$ & $0.110^{\star}$ & $0.120 *$ \\
\hline \multirow{2}{*}{$\begin{array}{l}\text { Model 6: } \\
\text { model } 1 \text { - sex }\end{array}$} & No censor & $0.133^{*}$ & $0.135^{\star}$ & $0.136^{\star}$ & $0.129 *$ \\
\hline & Censor & $0.109 *$ & $0.121^{*}$ & 0.122 & $0.122^{\star}$ \\
\hline \multirow{2}{*}{$\begin{array}{l}\text { Model 7: } \\
\text { model } 1 \text { - AVAT }\end{array}$} & No censor & $0.134^{*}$ & $0.128^{*}$ & $0.127 *$ & $0.122^{\star}$ \\
\hline & Censor & $0.112^{\star}$ & $0.105^{\star}$ & $0.099 *$ & $0.107^{*}$ \\
\hline \multirow{2}{*}{$\begin{array}{l}\text { Model 8: } \\
\text { model } 1 \text { - AVSI }\end{array}$} & No censor & $0.132^{*}$ & $0.132^{\star}$ & $0.129 *$ & $0.127^{*}$ \\
\hline & Censor & $0.109 *$ & $0.110 *$ & 0.103 & $0.118^{*}$ \\
\hline \multirow{2}{*}{$\begin{array}{l}\text { Model 9: } \\
\text { model } 1 \text { - cash margin }\end{array}$} & No censor & $0.133^{*}$ & $0.120^{\star}$ & $0.118^{\star}$ & $0.117^{\star}$ \\
\hline & Censor & $0.110^{\star}$ & $0.104^{\star}$ & 0.096 & $0.115^{\star}$ \\
\hline \multirow{2}{*}{$\begin{array}{l}\text { Model 10: } \\
\text { model } 1 \text { - smoking }\end{array}$} & No censor & $0.137^{*}$ & $0.126^{\star}$ & $0.125^{\star}$ & $0.121^{*}$ \\
\hline & Censor & $0.115^{\star}$ & $0.115^{\star}$ & $0.108^{*}$ & $0.120^{\star}$ \\
\hline \multirow{2}{*}{$\begin{array}{l}\text { Model 11: } \\
\text { model } 1 \text { - alcohol intake }\end{array}$} & No censor & $0.129 *$ & $0.125^{\star}$ & $0.128^{\star}$ & $0.125^{\star}$ \\
\hline & Censor & $0.107 *$ & $0.111^{\star}$ & $0.111^{*}$ & $0.124^{\star}$ \\
\hline \multirow{2}{*}{$\begin{array}{l}\text { Model 12: } \\
\text { model } 1 \text { - obesity }\end{array}$} & No censor & $0.135^{\star}$ & $0.132^{\star}$ & $0.131^{\star}$ & $0.130 *$ \\
\hline & Censor & $0.115^{\star}$ & 0.118 & 0.115 & $0.125^{\star}$ \\
\hline \multirow{2}{*}{ Model 13: significant terms in full model ${ }^{c}$} & No censor & $0.129 *$ & $0.123^{*}$ & $0.123^{\star}$ & $0.120^{*}$ \\
\hline & Censor & $0.113^{*}$ & $0.103^{*}$ & $0.103^{\star}$ & $0.101 *$ \\
\hline \multirow[t]{2}{*}{ Model 14: model 13 - education level } & No censor & $0.121 *$ & $0.127^{*}$ & $0.125^{\star}$ & $0.126^{*}$ \\
\hline & Censor & $0.105^{\star}$ & $0.107^{\star}$ & $0.107^{*}$ & $0.107 *$ \\
\hline \multirow[t]{2}{*}{ Model 15: model 13 - marital status } & No censor & $0.136^{*}$ & $0.133^{*}$ & $0.133^{\star}$ & $0.130 *$ \\
\hline & Censor & $0.116^{\star}$ & $0.107^{\star}$ & $0.106^{\star}$ & $0.105^{\star}$ \\
\hline \multirow[t]{2}{*}{ Model 16: model 13 - previous health } & No censor & $0.159 *$ & $0.154^{*}$ & $0.152^{*}$ & $0.153^{*}$ \\
\hline & Censor & $0.132^{\star}$ & $0.121^{\star}$ & $0.121^{\star}$ & $0.120^{*}$ \\
\hline \multicolumn{6}{|c|}{ * $\mathrm{p}<0.05$ based on the $2.5 \%$ and 97.5 Bootstrap percentiles. } \\
\hline \multicolumn{6}{|c|}{ a Participants were censored $(n=620)$ or not censored $(n=805)$ for unemployment during the follow-up period between 1995 and 2007.} \\
\hline \multicolumn{6}{|c|}{$\begin{array}{l}{ }^{b} \text { Full model controlling for alcohol intake, Availability of Attachment (AVAT), Availability of Social Integration (AVSI), body mass index, cash margin, } \\
\text { education level, gender, marital status, previous health status, occupation and smoking. }\end{array}$} \\
\hline \multicolumn{6}{|c|}{ c The significant variables in model 13-24 were education level, marital status, previous health status and occupation. } \\
\hline \multicolumn{6}{|c|}{$\begin{array}{l}\text { IPW }=\text { inverse probability weighting estimator. Estimates represent the health effect on unemployed compared to employed individuals where estimate } \\
\text { above } 0 \text { is poorer health for unemployed. Note: "- means that this variable was excluded in analyses compared to either model } 1 \text { or } 13 \text { and " }+ \text { " means tha } \\
\text { the variable was added to the significant variables in the analyses. }\end{array}$} \\
\hline
\end{tabular}




\begin{tabular}{|c|c|c|c|c|c|}
\hline \multirow[t]{2}{*}{ Model 17: model 13 -occupation } & No censor & $0.136^{*}$ & $0.123^{\star}$ & $0.123^{*}$ & $0.122 *$ \\
\hline & Censor & $0.116^{\star}$ & $0.105^{\star}$ & $0.104^{*}$ & $0.102^{\star}$ \\
\hline \multirow[t]{2}{*}{ Model 18: model $13+$ sex } & No censor & $0.130 *$ & $0.118^{*}$ & $0.117^{\star}$ & $0.116^{\star}$ \\
\hline & Censor & $0.114^{\star}$ & 0.094 & 0.093 & 0.096 \\
\hline \multirow[t]{2}{*}{ Model 19: model $13+$ AVAT } & No censor & $0.129 *$ & $0.123^{*}$ & $0.122^{\star}$ & $0.121^{\star}$ \\
\hline & Censor & $0.112^{\star}$ & $0.107 *$ & $0.107 *$ & $0.107 *$ \\
\hline \multirow[t]{2}{*}{ Model 20: model 13 + AVSI } & No censor & $0.131^{\star}$ & $0.122^{\star}$ & $0.122^{*}$ & $0.118^{*}$ \\
\hline & Censor & $0.115^{\star}$ & $0.105^{\star}$ & $0.105^{\star}$ & $0.102^{\star}$ \\
\hline \multirow[t]{2}{*}{ Model 21: model $13+$ cash margin } & No censor & $0.131^{\star}$ & $0.132^{\star}$ & $0.132^{*}$ & $0.130 *$ \\
\hline & Censor & $0.115^{\star}$ & $0.115^{\star}$ & $0.114^{*}$ & $0.111^{\star}$ \\
\hline \multirow[t]{2}{*}{ Model 22: model 13 + smoking } & No censor & $0.126^{\star}$ & $0.126^{\star}$ & $0.128^{*}$ & $0.126^{\star}$ \\
\hline & Censor & $0.109 *$ & $0.106^{*}$ & $0.107^{*}$ & $0.106^{*}$ \\
\hline \multirow[t]{2}{*}{ Model 23: model 13 + alcohol intake } & No censor & $0.132^{\star}$ & $0.127 *$ & $0.126^{*}$ & $0.122^{\star}$ \\
\hline & Censor & $0.115^{\star}$ & $0.105^{\star}$ & $0.105^{\star}$ & $0.102^{*}$ \\
\hline \multirow[t]{2}{*}{ Model 24: model 13 + obesity } & No censor & $0.129 *$ & $0.122^{*}$ & $0.122^{*}$ & $0.118^{\star}$ \\
\hline & Censor & $0.110^{\star}$ & 0.097 & 0.097 & 0.096 \\
\hline \multicolumn{6}{|c|}{ * $p<0.05$ based on the $2.5 \%$ and 97.5 Bootstrap percentiles. } \\
\hline \multicolumn{6}{|c|}{ a Participants were censored $(n=620)$ or not censored $(n=805)$ for unemployment during the follow-up period between 1995 and 2007.} \\
\hline \multicolumn{6}{|c|}{$\begin{array}{l}\text { b Full model controlling for alcohol intake, Availability of Attachment (AVAT), Availability of Social Integration (AVSI), body mass index, cash margin, } \\
\text { education level, gender, marital status, previous health status, occupation and smoking. }\end{array}$} \\
\hline \multicolumn{6}{|c|}{ c The significant variables in model 13-24 were education level, marital status, previous health status and occupation. } \\
\hline
\end{tabular}

For the self-reported long-term unemployment definition with no censoring, there were statistically significant odds ratios for all 24 models, with some variation dependent on the included variables, with odds ratios ranging between 1.77 and 2.00 for unemployment compared with employment (Table 3 ). The highest odds ratios occurred when previous health was removed in model 16 , but still not higher than the crude odds ratio (2.06). For the definition with censoring, the odds ratios ranged between 1.66 and 1.88 for the different models. The crude odds ratio (1.78) was within this range. Of odds ratios other than the one for labour market status, mainly occupation and education affected each other when one of them was removed. The odds ratios then increased, in some cases even shifting to non-significance. The exclusion of previous health affected the odds ratios of some variables, including $0.03-0.15$ lower odds ratio for labour market status.

For the risk difference estimators, there was an absolute increase in poor health due to unemployment that ranged from $9-16 \%$, most of which was statistically significant, depending on the model and estimator (Table 4). Removal or addition of variables had some effect on estimates, where the largest effects resulted mainly from the removal of previous health from the confounders. When models were alternated with non-significant terms in the full model, the largest deviation was 0.014 for the risk difference (model 6 for censoring during follow-up) in comparison with model 1 or model 13 . The deviation between models was at most 0.005 under this situation for the G-computation estimator.

\section{Discussion}

In our study, we have shown that the unemployment mode has a considerable effect on estimates of the health effect of unemployment. It was interesting to notice that the absolute difference between self-reported and register-based unemployment was so substantial, ranging from as much as $2.5-3.1 \%$ for the risk difference estimators when there was no censoring for unemployment episodes during the follow-up period, and even greater when censoring for unemployment episodes. The results were similar for the logistic regression estimator too.

On the other hand, our evaluation of assumptions in the statistical models yielded only rather marginal deviations in most cases. The exceptions were mainly related to the doubly-robust estimator, which is known to be sensitive to small samples, where we were able to identify some notable differences between the full model with all its variables and the model with only significant potential confounders, and estimates based on the current unemployment mode. For other comparisons between these models, the difference was no greater than $1.1 \%$, and in most cases it was at most $0.6 \%$.

Also, in the models where we evaluated the contribution of each potential confounder to the estimates, by either adding or removing a variable to or from the full or the reduced model, the effect estimates were at most marginally affected, with the exception of the presence of previous health in the analyses. Thus, 
our study shows that even if a correctly specified model is advantageous, the risk of bias is likely to be rather limited for similar research questions such as those we have investigated. To avoid biases due to the model set-up, it is paramount to take health selection in unemployment into consideration.

It is interesting that the more popular logistic regression estimator seems to be more sensitive to poor model assumptions than the propensity scores and Gcomputation methods. The comparison of our risk difference estimators, which showed that they yield mainly similar results, is very much in line with previous comparisons between propensity score methods and conventional multivariable methods [19]. Thus, it seems that the choice of the statistical method is not the main challenge in achieving estimates with a low bias.

Even if our study indicates that the statistical model might only cause small biases, the importance of the choice of variables is undoubtedly very important. Interestingly, in a review from 2014, only 6 of the 41 reviewed articles discussed the choice of statistical method, and if any of these publications discussed how to measure unemployment it was at least very rare [3]. Thus, among most researchers there is a need for a more informative way of describing potential limitations from the choice of statistical model.

Our interest was in the estimate for labour market status. Interestingly, when studying how other estimates were affected by the model set-up, we observed that education and occupation were in some cases not significant on their own, while they were significant in combination, while the estimate of labour market was not as sensitive to the presence of these variables in the analysis. This behaviour of the estimates for education and occupation is contrary to the expectations when a variable is added, as collinearity is expected to lead to less statistical significances than the other way round. This finding further highlights the importance of a well thought through variable selection in the main analyses. Nevertheless, the rationale for including variables in the statistical analysis needs to be improved as has been highlighted in previous research [3,20].

The importance of a correctly specified research question for the results was one of the key issues in our study. Whether unemployment is self-reported or taken from a register should respond to the same research question and yield similar results. In our study, the deviation between results for register-based and self-reported labour market was rather large. To some extent, this might be explained by the register data not being available until the day of the survey. We do however think that the main explanation for the deviation is that differences in how data are collected lead to slightly different research questions being answered.

Neither the accumulated unemployment spell during recent years nor current unemployment might cover how recent or current unemployment affects health in the long term. They may both be too limited in that the accumulated unemployment relates too much to historical unemployment and current to a very short and negligible unemployment spell for the person in question. Thus, it is likely to be more complicated to know how unemployment affects health, and, hence, also the importance of including health status at the time of unemployment into the analysis. The big discrepancy between the estimates from the measures used to collect unemployment in our study highlights the importance of a well thought-through measure. Thus, the main message in our work is to gain a deep understanding of how unemployment data are collected. Based on our experience, we recommend accumulated self-reported unemployment measured with a detailed retrospective matrix.

We have made a thorough analysis of different aspects of the analysis of unemployment. A possible limitation of our study is that only one population has been analysed and, despite different research questions used, we have still had a somewhat limited focus of the consequences of long-term unemployment on health. Even so, we believe that our results can have a major contribution for the future understanding of the priorities needed not only for this research topic but also for other research topics.

\section{Conclusions}

The choice of how to measure unemployment has had a rather large impact on effect estimates and if unemployment is incorrectly measured, and thus responding to a different research question, our study shows that results can vary substantially. There was even a notable deviation between results for register-based and self-reported unemployment, which probably did not depend only on the register not being able to capture unemployment until the day of the survey. In most cases, the choice of statistical method and variables in the model only had a very small effect on effect estimates, and only previous health needed to be handled well in the statistical analysis model. Still, researchers should put particular emphasis on model diagnostics and carefully evaluate whether the assumptions made in their analyses hold true and, more importantly, that any assumptions made are carefully taken care of, and better discussed and motivated in publications.

\section{Abbreviations}

AVAT - Availability of attachment instrument

AVSI - Availability of social integration instrument

BMI - Body mass index

IPW - inverse probability weighting

LISA - the longitudinal integration database for health insurance and labour market studies

MSE - mean square error

\section{Declarations}




\section{Ethics approval and consent to participate}

The Regional Ethical Board in Umeå, Sweden, approved the study.

Informed consent to the study was given by the participants by returning their questionnaire. The study, and the methods used, were carried out in accordance with relevant guidelines and regulations. In the supplementary file, we have document this, following the STROBE guideline.

\section{Consent for publication}

Not applicable.

\section{Availability of data and material}

The questionnaires that were used for this study are available at https://ki.se/en/imm/the-northern-swedish-cohort. The datasets generated and/or analyzed during the current study are not publicly available because the Swedish Data Protection Act (1998:204) does not permit sensitive data on humans (like in our interviews) to be freely shared. The datasets are available based on ethical permission from the Regional Ethical board in Umeå, Sweden, from one of the coauthors (Anne Hammarström).

\section{Competing interests}

The authors declare that they have no competing interests.

\section{Funding}

The study was funded by the Swedish Research Council for Health, Working Life and Welfare (dnr 2011-0839). The funder had no role in the design of the study and collection, analysis, and interpretation of data and in writing the manuscript.

\section{Authors' contributions}

The study was designed by FN in collaboration with $\mathrm{AH}$. FN performed the analyses and the interpretations in collaboration with AH. FN drafted the paper, and AH contributed actively. All authors read and approved the final manuscript.

\section{Acknowledgements}

The authors would like to thank all of the participants of the study. The authors would like to thank Umeå University for collaboration around the Northern Sweden cohort.

\section{References}

1. Norström F, Virtanen P, Hammarström A, Gustafsson P, Janlert U: How does unemployment affect self-assessed health? A systematic review focusing on subgroup effects. BMC Public Health 2014, 14(1):1310.

2. Paul KI, Moser K: Unemployment impairs mental health: Meta-analyses. Journal of Vocational Behavior 2009, 74(3):264-282.

3. Norström F: Poor quality in the reporting and use of statistical methods in public health - the case of unemployment and health. Archives of Public Health 2015, 73:56.

4. Lucena C, Lopez JM, Pulgar R, Abalos C, Valderrama MJ: Potential errors and misuse of statistics in studies on leakage in endodontics. International Endodontic Journal 2013, 46(4):323-331.

5. Stauder J: Unemployment, unemployment duration, and health: selection or causation? The European Journal of Health Economics 2019, 20(1):59-73.

6. Bagley SC, White H, Golomb BA: Logistic regression in the medical literature: Standards for use and reporting, with particular attention to one medical domain. Journal of clinical epidemiology 2001, 54(10):979-985.

7. Rosenbaum PR, Rubin DB: The Central Role of the Propensity Score in Observational Studies for Causal Effects. Biometrika 1983, 70(1):41-55.

8. Snowden JM, Rose S, Mortimer KM: Implementation of G-Computation on a Simulated Data Set: Demonstration of a Causal Inference Technique. American Journal of Epidemiology 2011, 173(7):731-738.

9. Norström F, Janlert U, Hammarström A: Is unemployment in young adulthood related to self-rated health later in life? Results from the Northem Swedish cohort. BMC Public Health 2017, 17(1):529.

10. Shah BR, Laupacis A, Hux JE, Austin PC: Propensity score methods gave similar results to traditional regression modeling in observational studies: a systematic review. Journal of clinical epidemiology 2005, 58(6):550-559.

11. Hammarström A, Janlert U: Cohort profile: the northern Swedish cohort. Int J Epidemiol 2012, 41(6):1545-1552. 
12. Virtanen P, Lipiainen L, Hammarström A, Janlert $U$, Saloniemi A, Nummi T: Tracks of labour market attachment in early middle age: A trajectory analysis over 12 years. Adv Life Course Res 2011, 16(2):55-64.

13. Socioekonomisk indelning (SEI) [http://www.scb.se/statistik/_publikationer/OV9999_1982A01_BR_X11\%C3\%96P8204.pdf]

14. Henderson S, Duncan-Jones P, Byrne DG, Scott R: Measuring social relationships. The Interview Schedule for Social Interaction. Psychol Med 1980, 10(4):723-734.

15. Brookhart MA, Wyss R, Layton JB, Sturmer T: Propensity Score Methods for Confounding Control in Nonexperimental Research. CirculationCardiovascular Quality and Outcomes 2013, 6(5):604-611.

16. Lunceford JK, Davidian M: Stratification and weighting via the propensity score in estimation of causal treatment effects: a comparative study. Statistics in Medicine 2004, 23(19):2937-2960.

17. Davison AC, Hinckley DV: Bootstrap Methods and their Application. Cambridge, United Kingdom: Cambridge University Press; 1997.

18. R Core Team: R: A Language and Environment for Statistical Computing. R Foundation for Statistical Computing; 2015.

19. Sturmer T, Joshi M, Glynn RJ, Avorn J, Rothman KJ, Schneeweiss S: A review of the application of propensity score methods yielded increasing use, advantages in specific settings, but not substantially different estimates compared with conventional multivariable methods. Journal of Clinical Epidemiology 2006, 59(5):437-447.

20. Pouwels KB, Widyakusuma NN, Groenwold RH, Hak E: Quality of reporting of confounding remained suboptimal after the STROBE guideline. J Clin Epidemiol 2015.

\section{Supplementary Files}

This is a list of supplementary files associated with this preprint. Click to download.

- STROBEchecklistBMCcrosssectionalstudies211125.docx 